\title{
Specific Heat of Disordered Superconductors Induced by Negative Centers
}

\author{
Grzegorz Litak ${ }^{1}$ \\ Department of Mechanics, Technical University of Lublin, Ul. Nadbystrzycka 36, PL-20-618 Lublin, Poland. \\ Max Planck Institute for Physics of Complex Systems, Nöthnitzer Str. 38, D-01187 Dresden, Germany.
}

\begin{abstract}
We show that superconductors with inhomogeneous order parameters can show similar features as anisotropic ones. In this paper we study the low temperature specific heat dependence in such a system and we show that the disorder associated with randomly distributed attractive centers convert the BCS temperature exponential behaviour into a power law formula. To describe superconductivity we used a random version of the negative U Hubbard model, while the disorder was treated by means of the Coherent Potential Approximation (CPA).
\end{abstract}

Key words: superconductivity, doping, fluctuations, specific heat PACS: 74.62.Dh, 74.40.+k, 74.25.Bt

According to the Anderson theorem the effect of non-magnetic disorder on s-wave superconductors can be neglected unless spatial fluctuations of the order parameter are negligible $[1,2]$. This theorem for conventional superconductors can also be applied to other superconductors with a long coherence length where the fluctuations of the order parameter induced by disorder $[2,3]$ are small and the quasiparticle density of states shows a clear gap. As a consequence, superconducting properties, such as the specific heat and the magnetic penetration depth, feature the typical exponential BCS dependences. The situation is quite different for exotic superconductors. There the order parameter is modulated by a wave vector $\mathbf{k}$ and even

\footnotetext{
1 Tel.: +48- 81- 5381573; Fax: +48- 81- 5241004; E-mail: litak@archimedes.pol.lublin.pl
}

disappears (for d-wave symmetry of the order parameter) at Fermi surface line nodes.

In the case of the recently discovered $\mathrm{MgB}_{2}$ [4] with a high critical temperature $T_{C} \approx 40 \mathrm{~K}$ and a relatively short coherence length $\xi$ (from 25 to $120 \AA$, depending on the sample [5]) the Anderson assumption of a uniform order parameter is also not satisfied. In fact, there is a lot of evidence [5] that in this compound the multi-gap scenario with a spatially fluctuating s-wave order parameter is realized. Moreover Sharma et al. [6] found a percolative transition of superconductivity in $\mathrm{Mg}_{1-x} \mathrm{~B}_{2}$.

Prompted by these findings we examine the effects of a low temperature specific heat dependence using the random negative U Hubbard model proposed to describe percolation in superconductors [7]. We study the case where $U_{i}$ is $-|U|$ and 0 with probability $c$ and $1-c$, respectively on a square 
lattice whose sites are labelled $i$ using the Gorkov decoupling [8] and the Coherent Potential Approximation (CPA) [7].

(a)

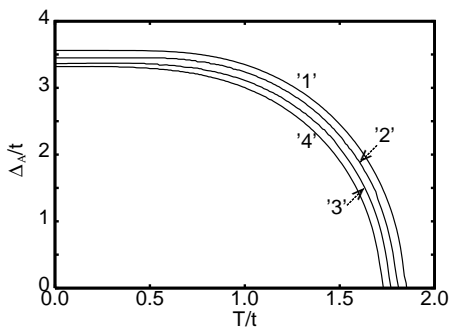

(b)

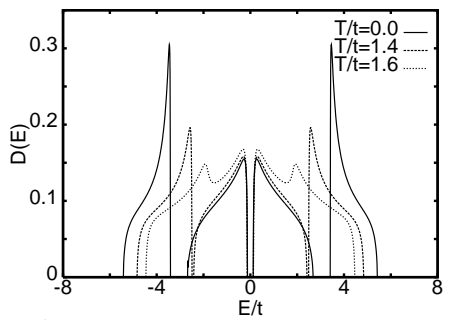

(c)

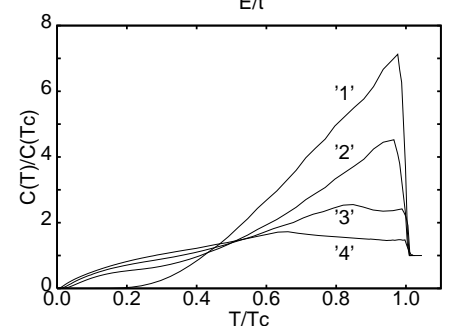

Fig. 1. Temperature dependence of $\Delta_{A}$ for various concentrations of negative centers $c$ in a binary alloy $\mathrm{A}_{c} \mathrm{~B}_{1-c}(\mathrm{a})$. Quasiparticle density of states for $c=0.5$ and three different temperatures (b). Specific heat for superconductors with various concentration of negative centers (c). In Figs. 1 (a) and (c) lines denoted by '1-4' correspond to $c=0$, $0.7,0.5,0.3$, respectively.

For the above assumptions we have done calculations of the order parameter $\Delta_{A}$ for various concentrations of negative centers $c$ in a binary alloy $\mathrm{A}_{c} \mathrm{~B}_{1-c}$ [7]. Here, for simplicity, we neglected the Hartree term $U n / 2$. This term may be important in some other contexts, as it is known to lead to a band splitting phenomenon and consequently to the appearance of the critical concentration of a superconducting phase $c_{0}[7]$.

The temperature dependences of $\Delta_{A}$ for $U_{A}=$ $-8 t$ are presented in Fig. 1a, while examples of quasiparticle densities of states (QDOS) for different temperatures are depicted in Fig. 1b. Note that the pairing potential $\Delta_{A}$ for $c \neq 1$ differs slightly from the uniform case $(c=1)$, however the QDOS with states inside the gap is fundamentally different.

Now the low temperature specific heat can be easily evaluated using the following formula $[5,9]$ :

$$
C=2 T \int_{-\infty}^{\infty} D(\omega) \frac{\omega}{T} \frac{\mathrm{d}}{\mathrm{d} T}\left(\frac{1}{\mathrm{e}^{\omega / T}+1}\right),
$$

where $D(\omega)$ denotes QDOS.

Fig. 1c shows the results for the specific heat for $c=1.0,0.7,0.5$ and 0.3 . Note that, in contrast to a clean case ('1') where the corresponding plot can be associated with exponential formula $C / T \sim$ $\exp (-\alpha / T)$ for constant $\alpha$, the next curves ('2-4') mimic a distinct power law behaviour (excluding some lowest temperatures).

Thus, the behaviour of superconductors with randomly distributed negative centers showing fluctuations of the pairing potential in real space resembles, in some sense, the behaviour of anisotropic but clean systems with a pairing potential varying in $\mathbf{k}$ space. Namely, a wave vector modulation of the superconducting order parameter in the case of s-wave and most of all line nodes in the case of d-wave superconductors lead to similar effects with a low temperature unusual dependence of the specific heat.

This work has been partially supported by the KBN Grant No. 3P03B06225.

\section{References}

[1] P.W. Anderson, J. Phys. Chem. Solids 11 (1959) 26.

[2] B.L. Györffy et al., in Fluctuation Phenomena in High Critical Temperature Superconducting Ceramics Eds. M. Ausloos and A.A. Varlamov (Kluwer Academic Publishers NATO ASI Series, Dordrecht 1997) 385396.

[3] R. Moradian et al., Phys. Rev. B 63 (2001) 024501.

[4] J. Nagamatsu et al., Nature 410 (2001) 63.

[5] A.M. Gabovich at al., J. Phys.: Condens. Matter 14 (2002) 9621.

[6] P.A. Sharma et al., Phys. Rev. Lett. 89 (2003) 167003.

[7] G. Litak, B.L. Györffy, Phys. Rev. B 62 (2000) 6629. 
[8] R. Micnas et al. Rev. Mod. Phys. 62 (1990) 113.

[9] B.L. Györffy et al., Phys. Rev. B 58 (1998) 1025. 